\title{
Case Report \\ Efficacy of Rituximab in a Systemic Lupus Erythematosus Patient Presenting with Diffuse Alveolar Hemorrhage
}

\author{
Gabriela Montes-Rivera, Grissel Ríos, and Luis M. Vilá \\ Division of Rheumatology, Department of Medicine, University of Puerto Rico Medical Sciences Campus, San Juan, PR, USA \\ Correspondence should be addressed to Luis M. Vilá; luis.vila2@upr.edu
}

Received 26 July 2017; Revised 17 October 2017; Accepted 22 October 2017; Published 15 November 2017

Academic Editor: Jamal Mikdashi

Copyright (c) 2017 Gabriela Montes-Rivera et al. This is an open access article distributed under the Creative Commons Attribution License, which permits unrestricted use, distribution, and reproduction in any medium, provided the original work is properly cited.

Diffuse alveolar hemorrhage (DAH) is a life-threatening complication of systemic lupus erythematosus (SLE). Although infrequent, its mortality is very high. While there are no established therapeutic guidelines, DAH has been traditionally managed with high-dose intravenous (IV) corticosteroids, cyclophosphamide, and plasma exchange. The efficacy of alternative therapies such as rituximab has been described only in a few cases. Herein, we report a 25-year-old Hispanic man who presented with acuteonset SLE manifested by polyarthralgia, nephritis, seizures, pancytopenia, severe hypocomplementemia, and elevated anti-dsDNA antibodies. His disease course was complicated by DAH. His condition was refractory to high-dose intravenous (IV) methylprednisolone pulses, IV cyclophosphamide, and plasmapheresis. Given the lack of clinical response, he was started on IV rituximab $375 \mathrm{mg} / \mathrm{m}^{2}$ weekly for a total of four courses. He rapidly improved after the first two doses. Over the next seven months, he did not present recurrent pulmonary symptoms. Follow-up chest computed tomography did not show residual abnormalities. This case, together with other reports, suggests that rituximab is an effective therapeutic option for DAH in SLE.

\section{Introduction}

Systemic lupus erythematosus (SLE) is an autoimmune systemic illness that may affect several organs, including the lungs. There is a myriad of pulmonary manifestations in SLE that ranges from pleuritis and pneumonitis to catastrophic diffuse alveolar hemorrhage (DAH) [1]. The latter is one of the most feared complications given its potentially high mortality rate [2]. Although DAH has a high morbidity and mortality, optimal management guidelines have yet to be established. Conventional treatment includes intravenous pulse (IV) corticosteroids, cyclophosphamide, and plasmapheresis [3]. The efficacy of alternative therapies for DAH in SLE such as rituximab has been described only in a few cases [4-17]. Herein, we report the case of a 25-year-old man with SLE with DAH who successfully responded to rituximab therapy.

\section{Case Presentation}

A 25-year-old man with no history of systemic illness was hospitalized on August 21, 2016, due to a 1-week history of fever, general malaise, polyarthralgia, abdominal discomfort, and diarrhea. Initial physical examination was unremarkable except for the presence of nasal ulcers and swelling of hands, ankles, and feet. Laboratory findings showed leukopenia ( white blood cell count $=2.9 \mathrm{~K} / \mathrm{uL}$ ), lymphopenia (lymphocyte count $=0.60 \mathrm{~K} / \mathrm{uL}$ ), anemia (hemoglobin $=11.5 \mathrm{~g} / \mathrm{dL}$ ), and thrombocytopenia (platelet count $=133 \mathrm{~K} / \mathrm{uL}$ ). Reticulocyte count was elevated at $7.7 \%$, but haptoglobulin levels were normal and Coomb's test was negative. Serum chemistries revealed elevated creatinine levels $(1.49 \mathrm{mg} / \mathrm{dL})$ and hypoalbuminemia $(2.4 \mathrm{~g} / \mathrm{dL})$. Urine analysis revealed proteinuria and hematuria (8-30 red blood cells/high power field). A 24-hour urine collection disclosed proteinuria of $2570 \mathrm{mg}$ and decreased creatinine clearance at $61.5 \mathrm{~mL} / \mathrm{min}$. Westergren sedimentation rate was elevated at $124 \mathrm{~mm} / \mathrm{hr}$. Anti-nuclear antibodies (ANA) were positive $(1: 160$, homogenous pattern), and anti-dsDNA antibodies were elevated $(>300 \mathrm{IU} / \mathrm{mL})$. He had marked C3 $(14 \mathrm{mg} / \mathrm{dL})$ and C4 $(<3 \mathrm{mg} / \mathrm{dL})$ hypocomplementemia. Anti-Ro, anti-La, anti-beta-2 glycoprotein 1 (IgA, IgG, and IgM), and anticardiolipin (IgA, IgG, and $\operatorname{IgM}$ ) antibodies were negative. 
The lupus anticoagulant test was negative. Viral hepatitis panel and HIV test were negative. Bone marrow biopsy, including immunophenotypic analysis, was negative for lymphoma or leukemia.

On August 29, 2016, the patient was treated with methylprednisolone 1 gram intravenous (IV) daily for 3 days, followed by $1 \mathrm{mg} / \mathrm{kg} /$ day of prednisone. Also, he was started on mycophenolate mofetil $500 \mathrm{mg}$ orally twice daily and hydroxychloroquine $200 \mathrm{mg}$ orally twice daily. On September 03, 2016, he developed hypertension $(168 / 111 \mathrm{~mm} \mathrm{Hg})$ and one episode of tonic-clonic seizures. Brain magnetic resonance imaging revealed changes consistent with posterior reversible encephalopathy syndrome. Brain magnetic resonance angiography was normal.

On September 08, 2016, he had a marked decrease of hemoglobin levels down to $8.7 \mathrm{~g} / \mathrm{dL}$. Chest computed tomography (CT) showed alveolar opacification and groundglass opacities of the right lung becoming near confluent in the central and lower lung. A similar opacification pattern was observed in the left lung. At this time, the patient had no respiratory distress or hemoptysis. Empiric antibiotics (cefepime and vancomycin) were added. Bronchoscopy performed on September 09, 2016, revealed pulmonary hemorrhage with the presence of hemosiderin-laden macrophages. On this day, prednisone was discontinued, and he was started on methylprednisolone $2 \mathrm{mg} / \mathrm{kg} /$ daily (methylprednisolone $60 \mathrm{mg}$ IV every $12 \mathrm{hr}$ ) and received cyclophosphamide $900 \mathrm{mg}$ IV. Mycophenolate mofetil was discontinued because of severe dyspepsia. Concurrently, plasmapheresis was started and given for five cycles. On September 10, 2016, the patient presented with bibasilar crackles, and hemoglobin levels dropped to $5.1 \mathrm{~g} / \mathrm{dL}$. Therefore, methylprednisolone dose was increased to $125 \mathrm{mg}$ IV every 12 hours. He persisted with hemoptysis and severe anemia which required several blood transfusions. After completing five cycles of plasmapheresis, he was treated again with methylprednisolone pulses $(500 \mathrm{mg}$ IV every 12 hours for 3 days). Despite these therapeutic interventions, the patient's condition continued to deteriorate, and on September 15, 2016, he required endotracheal intubation and mechanical ventilation. Hemoglobin levels decreased to $4.8 \mathrm{~g} / \mathrm{dL}$, and another course of plasmapheresis was given daily for seven days, then every other day for 10 days. In view that he continued with active pulmonary disease, rituximab $375 \mathrm{mg} / \mathrm{m}^{2}$ (690 mg) IV weekly for a total of four courses was started on September 16, 2016. Methylprednisolone dose was decreased to $125 \mathrm{mg}$ every 12 hours. On September 20, 2016, Klebsiella pneumoniae was detected on sputum culture for which polymyxin B was added. On September 23, 2016, seven days after first dose of rituximab, clinical improvement of hemoptysis was noted evidenced by less blood suctioned from the endotracheal tube and improved mechanical ventilator parameters. On that day, the second dose of rituximab was administered. On September 25 , 2016, the patient was successfully extubated, and thereafter his condition continued to improve. Methylprednisolone dose was decreased to $125 \mathrm{mg}$ IV daily, followed by methylprednisolone $80 \mathrm{mg}$ IV daily. On September 29 , 2016, only minimal hemoptysis was reported. The third dose of rituximab was given on October 01, 2016. Мycophenolate mofetil $500 \mathrm{mg}$ orally daily was restarted on October 02, 2016, and its dose was increased as tolerated to $2000 \mathrm{mg}$ orally daily. The last dose of rituximab was administered on October 07, 2016. On the next day, IV methylprednisolone was switched to prednisone $60 \mathrm{mg}$ orally daily. Follow-up chest X-ray revealed complete resolution of the bilateral patchy infiltrates. He was discharged home on prednisone $60 \mathrm{mg}$ orally daily, hydroxychloroquine $200 \mathrm{mg}$ orally daily, and mycophenolate mofetil $2000 \mathrm{mg}$ orally daily.

Over the next seven months, prednisone dose was gradually tapered down to $10 \mathrm{mg}$ daily. He was continued on mycophenolate mofetil and hydroxychloroquine. During this follow-up period, he did not present recurrent pulmonary symptoms. Leukopenia, anemia, and thrombocytopenia resolved. Creatinine levels decreased to normal levels $(0.99 \mathrm{mg} / \mathrm{dL})$. Proteinuria decreased to less than $1 \mathrm{~g}$ daily. Anti-dsDNA antibodies turned negative, and C3 and C4 increased to normal levels $(130 \mathrm{mg} / \mathrm{dL}$ and $31 \mathrm{mg} / \mathrm{dL}$, resp.). Chest CT done seven months later did not show any abnormalities.

\section{Discussion}

Diffuse alveolar hemorrhage is a rare but catastrophic complication of SLE that warrants rapid diagnosis and aggressive treatment. Bronchoscopy is the diagnostic gold standard for $\mathrm{DAH}$ given that imaging findings consisting of bilateral patchy infiltrates may also be found in infectious etiologies and acute respiratory distress syndrome [18]. Despite its high mortality, no management guidelines have been established, and often, patients are refractory to conventional therapy consisting of pulse corticosteroids, cyclophosphamide, and plasma exchange. Consequently, novel treatments such as rituximab have been attempted [4-17].

To the best of our knowledge, there are fifteen SLE cases complicated with DAH who have been treated with rituximab, including ours [4-17]. The demographic features, clinical manifestations, treatment, and outcomes of these patients are depicted in Table 1. Twelve of the described cases were women, and eleven, including our patient, were young with ages between 20 and 30 years. Ethnicity varied among reported cases; two were Hispanics including our patient. As already stated in the literature, most patients who develop $\mathrm{DAH}$ had renal involvement. Eleven patients, including our own, had lupus nephritis. Similar to our patient, the most common serological marker abnormalities at the beginning of DAH were elevated double-stranded DNA antibodies and hypocomplementemia.

Most reported SLE patients with DAH who received rituximab were initially treated with IV corticosteroids, and those with longer disease duration were already on maintenance therapy with oral corticosteroids [4-17]. Given that most of them had renal involvement before the onset of $\mathrm{DAH}$, four were already on mycophenolate mofetil, and five had previously received cyclophosphamide. In nine cases, including ours, the patients were facing life-threatening $\mathrm{DAH}$ and failed treatment with high-dose corticosteroids 


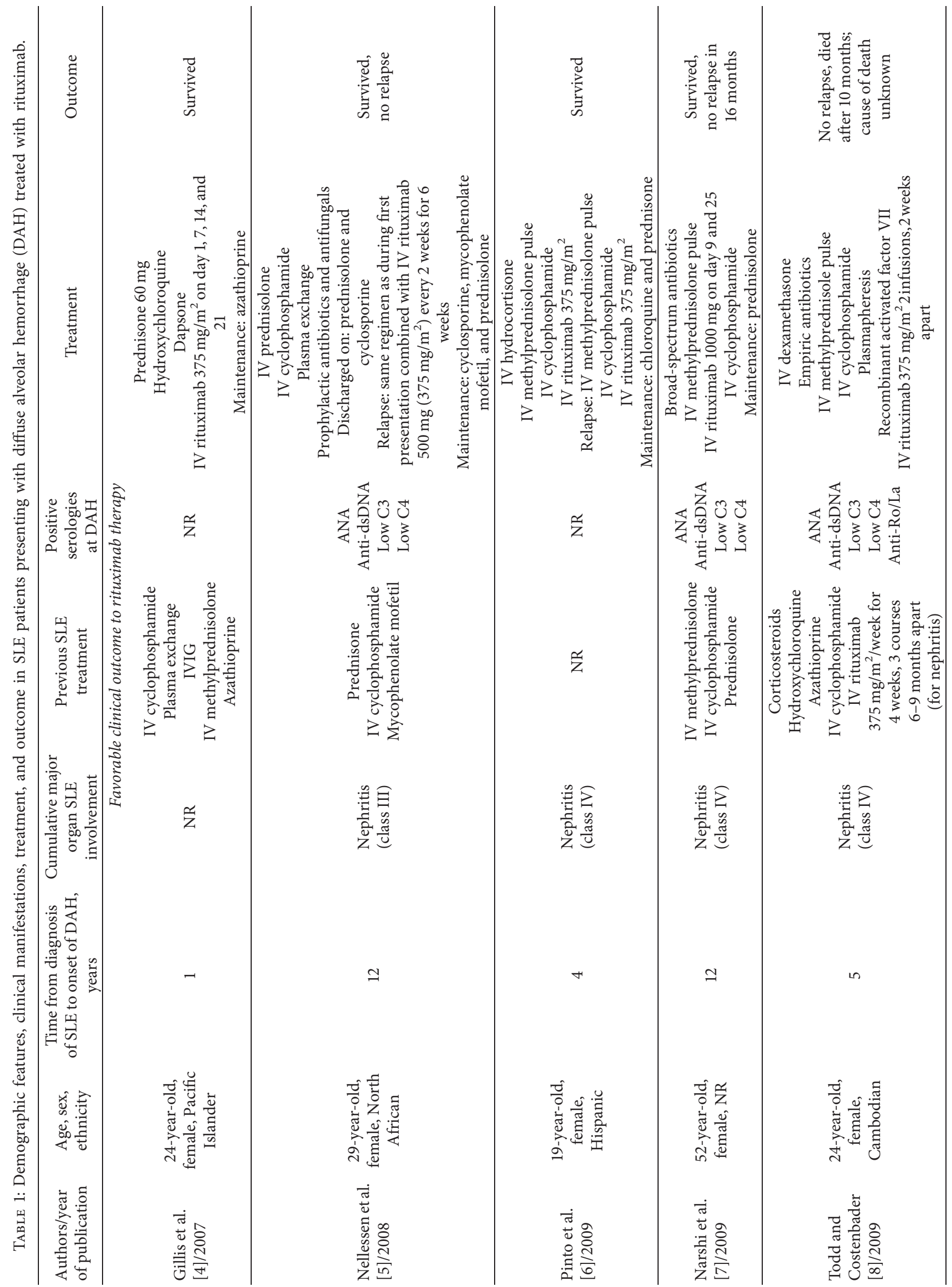




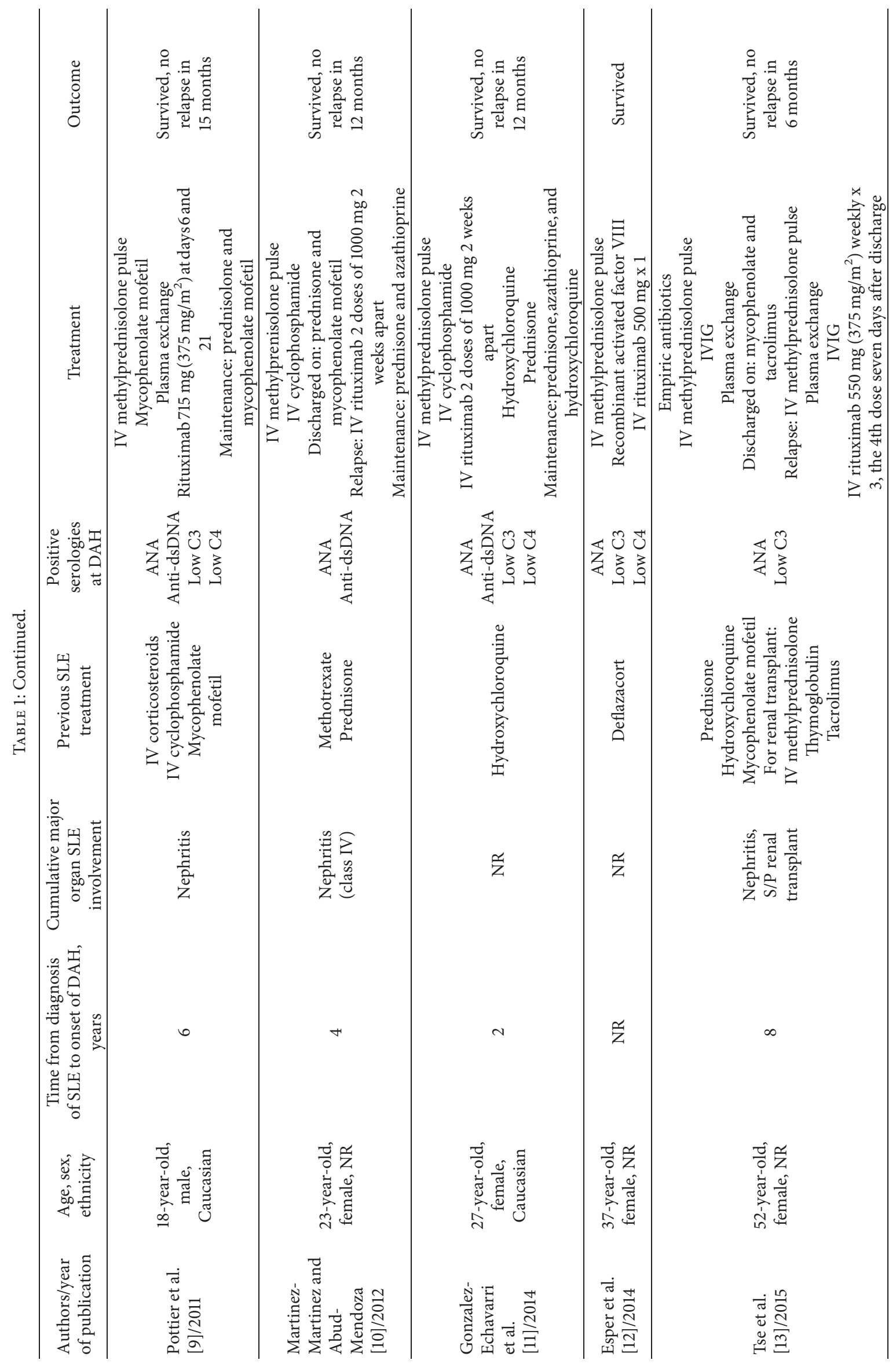




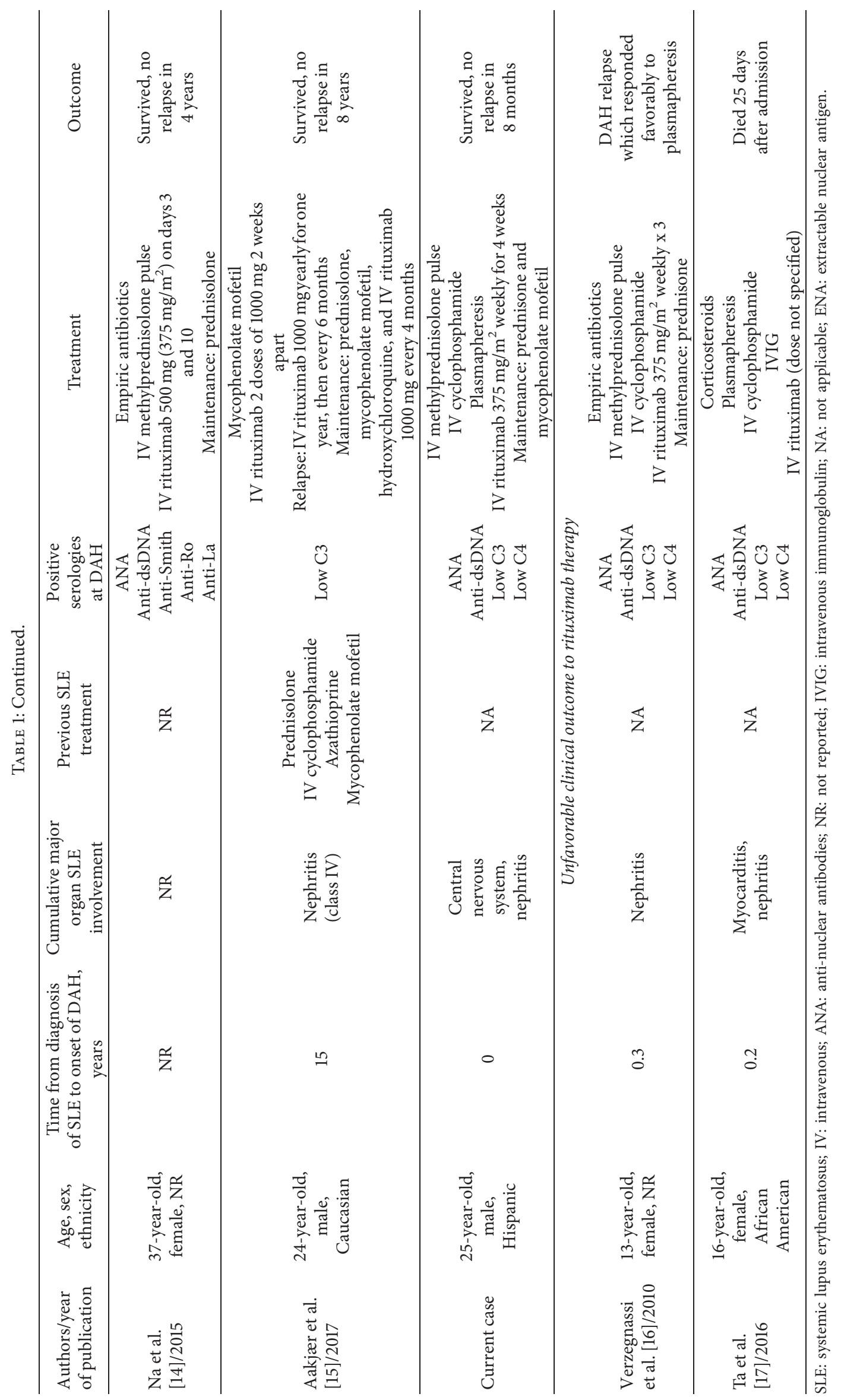


and cyclophosphamide. Six of the reported cases received plasma exchange. Thirteen out of fifteen patients successfully responded to rituximab, and in five cases, including our patient, the time of clinical improvement after rituximab was in less than two weeks. Noteworthy, six patients who improved with rituximab did not receive cyclophosphamide and/or pulse methylprednisolone therapy $[4,5,9,12,13,15]$.

Clinical outcomes of SLE patients presenting with DAH and treated with rituximab have been reported in case series. For example, Kazzaz et al. showed that three out of twentytwo patients who received rituximab, among other immunosuppressive treatments, survived [19]. On the other hand, in a case series of 140 SLE patients, three out of eight patients who received rituximab died [20]. However, in these case series, details about clinical manifestations, immunosuppressive treatments, and outcomes are not reported. Nonetheless, we have to acknowledge that cases with unfavorable outcomes are usually not reported with the same frequency as those with positive outcomes.

B-cell-depleting therapies are promising options for the management of SLE. Rituximab, an anti-CD20 monoclonal antibody, acts against the autoreactive $\mathrm{B}$ cells that regulate T-lymphocyte-dependent immune responses and dendritic cells [21]. Typically, its use in SLE is considered in refractory cases or even life-threatening situations such as severe lupus nephritis and myelitis and as a second- or third-line therapy in refractory cytopenias, vasculitis, and central nervous system involvement [22]. It can be argued that the clinical response observed in our patient was attributable to corticosteroids, plasmapheresis, and cyclophosphamide, and not to rituximab. However, the immunological changes after rituximab treatment occur early after infusion. A study conducted in lupus nephritis patients treated with rituximab showed that peripheral B cells were not detected by flow cytometry as quickly as one week after therapy in eight out of ten patients [23]. B-cell depletion was associated with a reduction in both ANA and dsDNA antibodies. Furthermore, in renal transplant patients, rituximab increases levels of IL-10 and MIP-1 $\beta$ after only 2 hours of drug infusion [24]. IL-10 is a known anti-inflammatory cytokine, and MIP- $1 \beta$ is a chemoattractant for regulatory $\mathrm{T}$ cells, thus inducing a downregulation of the immune response.

The potential role of rituximab for pulmonary hemorrhage is further supported by a murine model that showed the pivotal role of B cells in the pathogenesis of DAH by inducing lung inflammation with pristane $[25,26]$. This agent is a natural saturated terpenoid alkane that induces a lupus-like autoimmune syndrome. The bronchiolar lavage from pristane-treated mice showed the presence of monocytes and granulocytes as well as an increased number of CD19+ B cells and CD4+ and CD8+ T cells. These mice were more prone to develop alveolar hemorrhage. Conversely, mice that lacked both $B$ and T cells had significantly reduced frequency of DAH.

In summary, this case, together with other reports, suggests that rituximab might be an alternative drug for the treatment of DAH as it targets B-cell-mediated immune responses proven to be involved in the pathogenesis of this complication. Although frequently added to conventional therapies in refractory cases, the encouraging outcomes presented here set forth the possibility of designing controlled studies to examine the efficacy of rituximab in the induction phase.

\section{Conflicts of Interest}

The authors have no conflicts of interest to disclose.

\section{References}

[1] M. R. Zamora, M. L. Warner, R. Tuder, and M. I. Schwarz, "Diffuse alveolar hemorrhage and systemic lupus erythematosus. Clinical presentation, histology, survival, and outcome," Medicine, vol. 76, no. 3, pp. 192-202, 1997.

[2] A. S. Santos-Ocampo, B. F. Mandell, and B. J. Fessler, "Alveolar hemorrhage in systemic lupus erythematosus: presentation and management," Chest, vol. 118, no. 4, pp. 1083-1090, 2000.

[3] Y. Santiago-Casas and L. M. Vilá, "Pulmonary hemorrhage in patients with systemic lupus erythematosus," Current Respiratory Medicine Reviews, vol. 5, no. 1, pp. 49-54, 2009.

[4] J. Z. Gillis, M. Dall'era, A. Gross, J. Yazdany, and J. Davis, "Six refractory lupus patients treated with rituximab: a case series," Arthritis \& Rheumatism, vol. 57, no. 3, pp. 538-542, 2007.

[5] C. M. Nellessen, U. Pöge, K. A. Brensing, T. Sauerbruch, H. U. Klehr, and C. Rabe, "Diffuse alveolar haemorrhage in a systemic lupus erythematosus patient successfully treated with rituximab: a case report," Nephrology Dialysis Transplantation, vol. 23, no. 1, pp. 385-386, 2008.

[6] L. F. Pinto, L. Candia, P. Garcia et al., "Effective treatment of refractory pulmonary hemorrhage with monoclonal antiCD20 antibody (rituximab)," Respiration, vol. 78, no. 1, pp. 106-109, 2009.

[7] C. B. Narshi, S. Haider, C. M. Ford, D. A. Isenberg, and I. P. Giles, "Rituximab as early therapy for pulmonary haemorrhage in systemic lupus erythematosus," Rheumatology, vol. 49, no. 2, pp. 392-394, 2010.

[8] D. J. Todd and K. H. Costenbader, "Dyspnoea in a young woman with active systemic lupus erythematosus," Lupus, vol. 18, no. 9, pp. 777-784, 2009.

[9] V. Pottier, M. Pierrot, J. F. Subra et al., "Successful rituximab therapy in a lupus patient with diffuse alveolar haemorrhage," Lupus, vol. 20, no. 6, pp. 656-659, 2011.

[10] M. U. Martínez-Martínez and C. Abud-Mendoza, "Recurrent diffuse alveolar haemorrhage in a patient with systemic lupus erythematosus: long-term benefit of rituximab," Lupus, vol. 21, no. 10, pp. 1124-1127, 2012.

[11] C. Gonzalez-Echavarri, B. Pernas, A. Ugarte, and G. RuizIrastorza, "Severe multiorganic flare of systemic lupus erythematosus successfully treated with rituximab and cyclophosphamide avoiding high doses of prednisone," Lupus, vol. 23, no. 3, pp. 323-326, 2014.

[12] R. C. Esper, I. E. Estrada, T. de la Torre León, A. O. Gutiérrez, and J. A. López, "Treatment of diffuse alveolar hemorrhage secondary to lupus erythematosus with recombinant activated factor VII administered with a jet nebulizer," Journal of Intensive Care, vol. 2, no. 1, p. 47, 2014.

[13] J. R. Tse, K. E. Schwab, M. McMahon, and W. Simon, "Rituximab: an emerging treatment for recurrent diffuse alveolar hemorrhage in systemic lupus erythematosus," Lupus, vol. 24, no. 7, pp. 756-759, 2015.

[14] J. O. Na, S. H. Chang, K.-H. Seo et al., "Successful early rituximab treatment in a case of systemic lupus erythematosus 
with potentially fatal diffuse alveolar hemorrhage," Respiration, vol. 89, no. 1, pp. 62-65, 2015.

[15] S. Aakjær, E. Bendstrup, P. Ivarsen, and L. B. Madsen, "Continous rituximab treatment for recurrent diffuse alveolar hemorrhage in a patient with systemic lupus erythematosus and antiphosholipid syndrome," Respiratory Medicine Case Reports, vol. 22, pp. 263-265, 2017.

[16] F. Verzegnassi, F. Marchetti, F. Zennaro, A. Saccari, A. Ventura, and L. Lepore, "Prompt efficacy of plasmapheresis in a patient with systemic lupus erythematosus and diffuse alveolar haemorrhage," Clinical and Experimental Rheumatology, vol. 28, no. 3, pp. 445-446, 2010.

[17] R. Ta, R. Celli, and A. B. West, "Diffuse alveolar hemorrhage in systemic lupus erythematosus: histopathologic features and clinical correlations," Case Reports in Pathology, vol. 2017, Article ID 1936282, 6 pages, 2017.

[18] A. R. Lara and M. I. Schwarz, "Diffuse alveolar hemorrhage," Chest, vol. 137, no. 5, pp. 1164-1171, 2010.

[19] N. M. Kazzaz, P. Coit, E. E. Lewis, W. J. McCune, A. H. Sawalha, and J. S. Knight, "Systemic lupus erythematosus complicated by diffuse alveolar haemorrhage: risk factors, therapy and survival," Lupus Science \& Medicine, vol. 2, no. 1, p. e000117, 2015.

[20] C. Ednalino, J. Yip, and S. E. Carsons, "Systematic review of diffuse alveolar hemorrhage in systemic lupus erythematosus: focus on outcome and therapy," Journal of Clinical Rheumatology, vol. 21, no. 6, pp. 305-310, 2015.

[21] L. F. Pinto, C. J. Velásquez, C. Prieto, L. Mestra, E. Forero, and J. D. Márquez, "Rituximab induces a rapid and sustained remission in Colombian patients with severe and refractory systemic lupus erythematosus," Lupus, vol. 20, no. 11, pp. 1219-1226, 2011.

[22] M. Ramos-Casals, M. J. Soto, M. J. Cuadrado, and M. A. Khamashta, "Rituximab in systemic lupus erythematosus: a systematic review of off-label use in 188 cases," Lupus, vol. 18, no. 9, pp. 767-776, 2009.

[23] P. P. Sfikakis, J. N. Boletis, S. Lionaki et al., "Remission of proliferative lupus nephritis following B cell depletion therapy is preceded by down-regulation of the $\mathrm{T}$ cell costimulatory molecule CD40 ligand: an open-label trial," Arthritis \& Rheumatism, vol. 52, no. 2, pp. 501-513, 2005.

[24] E. G. Kamburova, M. W. F. van den Hoogen, H. J. P. M. Koenen, M. C. Baas, L. B. Hilbrands, and I. Joosten, "Cytokine release after treatment with rituximab in renal transplant recipients," Transplantation, vol. 99, no. 9, pp. 1907-1911, 2015.

[25] T. T. Barker, P. Y. Lee, K. M. Kelly-Scumpia et al., "Pathogenic role of B cells in the development of diffuse alveolar hemorrhage induced by pristane," Laboratory Investigation, vol. 91, no. 10, pp. 1540-1550, 2011.

[26] H. Zhuang, S. Han, P. Y. Lee et al., "Pathogenesis of diffuse alveolar hemorrhage in murine lupus," Arthritis \& Rheumatology, vol. 69, no. 6, pp. 1280-1293, 2017. 


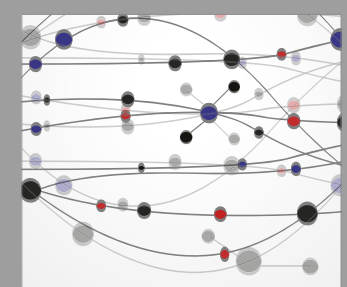

The Scientific World Journal
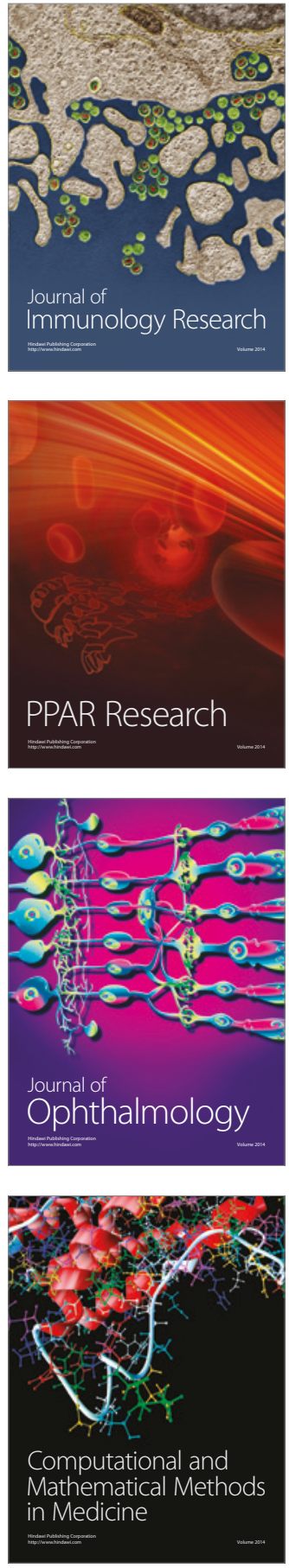

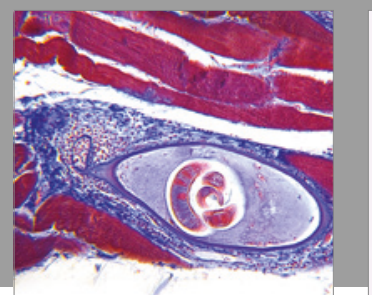

Gastroenterology Research and Practice
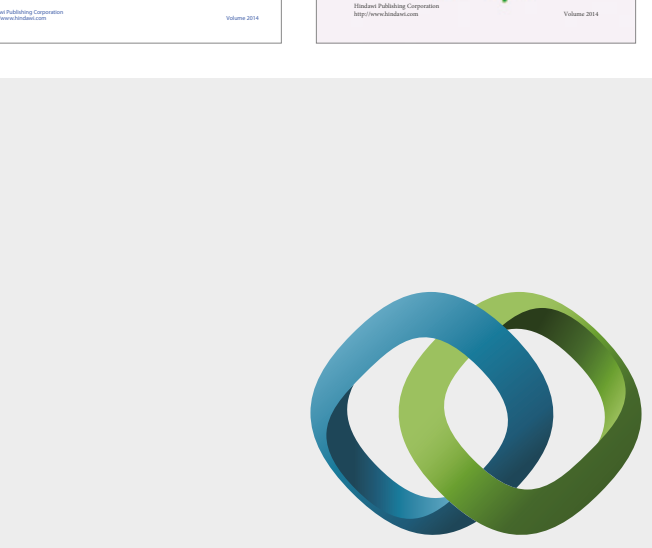

\section{Hindawi}

Submit your manuscripts at

https://www.hindawi.com
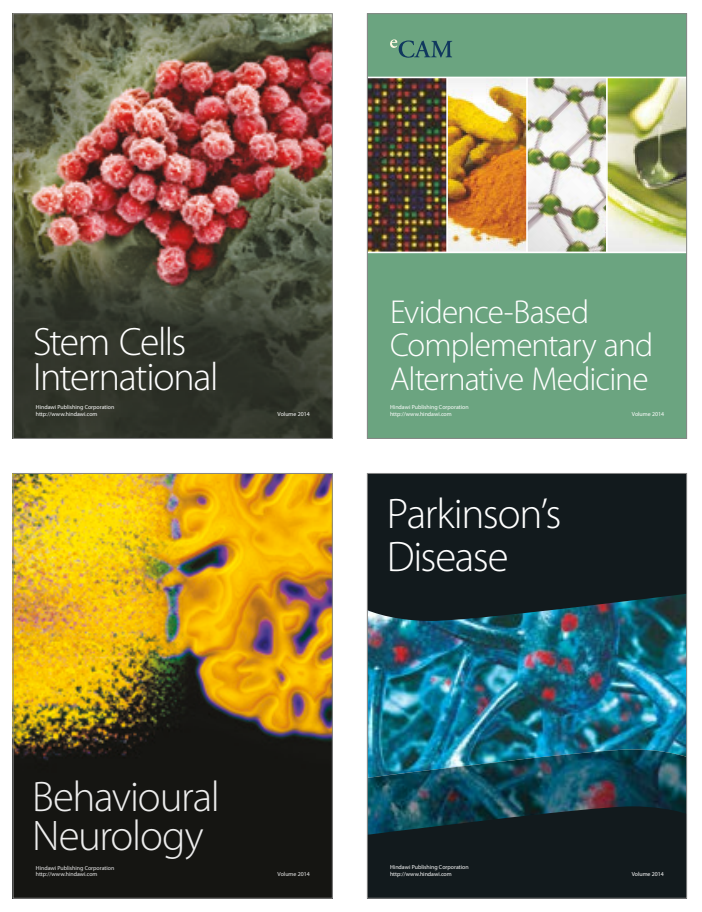
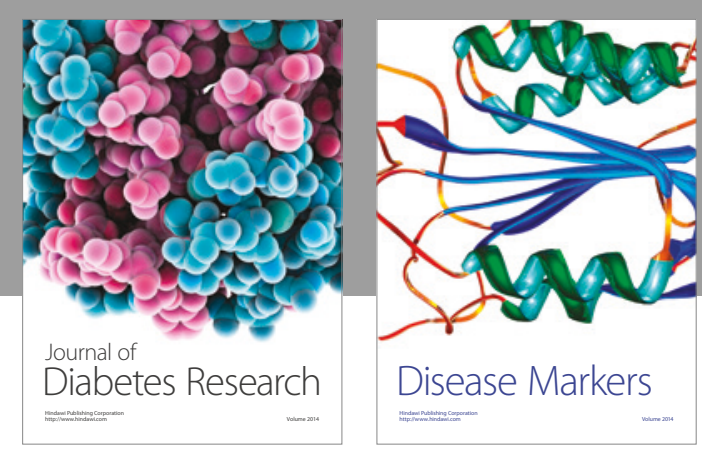

Disease Markers
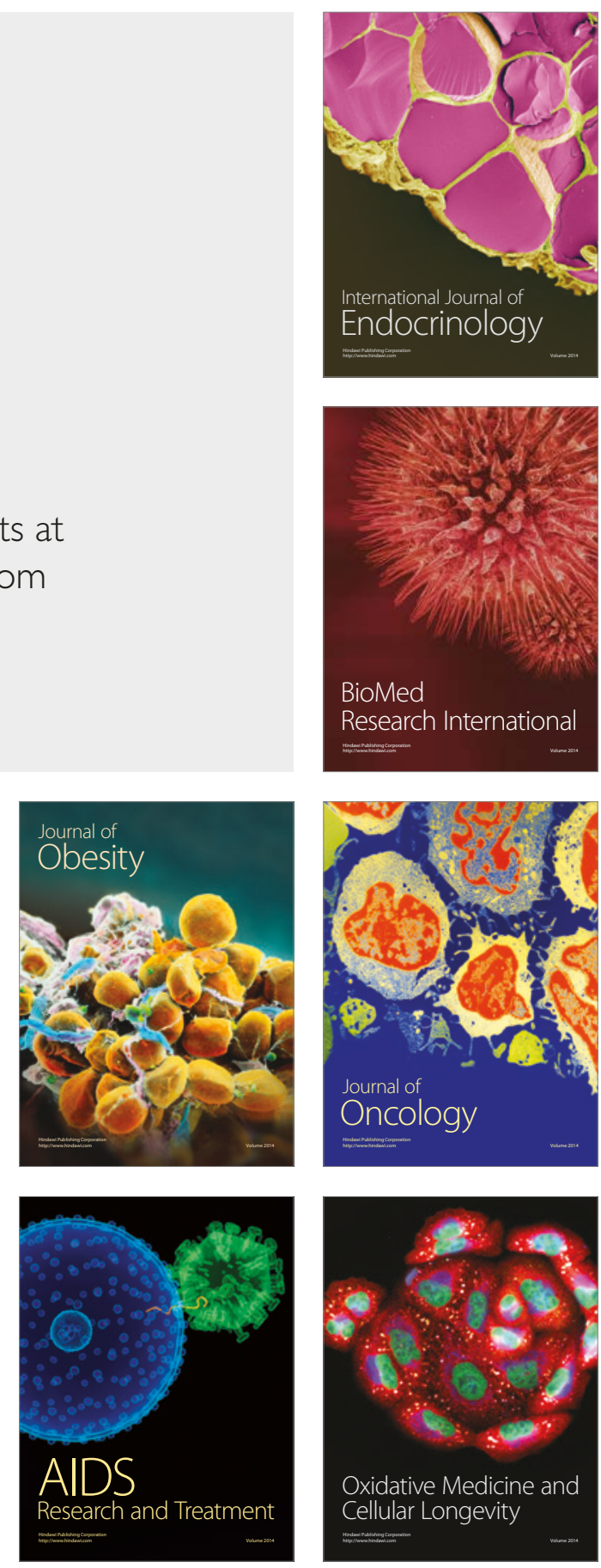